5th International Workshop on Astronomy and

Relativistic Astrophysics (IWARA2011)

International Journal of Modern Physics: Conference Series

Vol. 18 (2012) 109-114

(C) World Scientific Publishing Company

DOI: $10.1142 /$ S2010194512008306

\title{
ON THE TOPOLOGICAL NATURE OF THE COSMOLOGICAL CONSTANT
}

\author{
M. D. MAIA* \\ Institute of Physics, Universidade de Brasilia \\ 70910-97 Brasilia D.F. Brazil \\ maia@unb.br
}

\begin{abstract}
It is shown that topological changes in space-time are necessary to make General Relativity compatible with the Newtonian limit and to solve the hierarchy of the fundamental interactions. We detail how topology and topological changes appear in General Relativity and how it leaves an observable footprint in space-time. In cosmology we show that such topological observable is the cosmic radiation produced by the acceleration of the universe. The cosmological constant is a very particular case which occurs when the expansion of the universe into the vacuum occurs only in the direction of the cosmic time flow.
\end{abstract}

Keywords: Relativity, Groups, Topology.

\section{The Cosmological Constant Problem}

The cosmological constant $\Lambda$ was introduced by Einstein in 1917 to implement his static universe, but he removed it soon after, in face of the evidences for an expanding universe and the understanding that the presence of $\Lambda$ prevents the emergence of the Minkowski space-time as a particular solution of Einstein's gravitational equations. From the mathematical point of view, the cosmological constant originated from the contracted Bianchi identity $\left(R_{\mu \nu}-\frac{1}{2} R g_{\mu \nu}\right)_{; \rho} \equiv 0$. This conveys to the conclusion that the Einstein tensor (between the parenthesis) should be proportional to $\left(g_{\mu \nu}\right)_{; \rho} \equiv 0$ which is the defining condition for the Levi-Civita connection. Thus, $g_{\mu \nu}$ behaves as a constant under the metric covariant derivative. Since this same condition was already used for the derivation of the Einstein tensor, the identification of the contracted Bianchi identity to something proportional to $g_{\mu \nu}$ is a mathematical redundancy, using an argument that has been already spent.

Yet, the cosmological constant $\Lambda$ has been claimed to be the simplest explanation for the acceleration of the universe within the $\Lambda$ CDM paradigm. This follows from the derivation of the vacuum energy density of quantum fields ${ }^{1}$ as a solution of the

*Permanent Address 
semiclassical vacuum Einstein's equations ${ }^{\text {a }}$

$$
R_{\mu \nu}-\frac{1}{2} R g_{\mu \nu}-\Lambda g_{\mu \nu}=-8 \pi G<\rho_{v}>g_{\mu \nu}
$$

where $\left\langle\rho_{v}\right\rangle$ denotes the constant vacuum energy density. Since the evaluation of the energy density is made with the standard quantum field theory defined in Minkowski's space-time, then it must cancel exactly with $\Lambda$ :

$$
<\rho_{v}>=\Lambda / 8 \pi G
$$

so that the existence of the Minkowski space-time as a solution of the classical Einstein's equations would be guaranteed. However, the theoretical estimates of $\left\langle\rho_{v}>\right.$ gives $\left\langle\rho_{v}\right\rangle \approx 10^{76} \mathrm{GeV}^{2} / \mathrm{c}^{4}$. On the other hand, the acceleration of the universe indicates an experimental value of $\Lambda / 8 \pi G \approx 10^{-47} \mathrm{GeV}^{2} / \mathrm{c}^{4}$. This very large difference cannot be fixed within quantum field theory ${ }^{2,3}$. Consequently either from the geometrical or from the physical points of view, the presently accepted explanation for the acceleration of the universe is ill founded.

\section{Topology of Space-times}

Perhaps the least acknowledged fact about Einstein's gravitational theory is that it requires the notion of topological change to make it consistent with the presence of Newton's gravitational constant $G$ in Einstein's equations

$$
R_{\mu \nu}-\frac{1}{2} R g_{\mu \nu}=8 \pi G T_{\mu \nu}
$$

Newton's space-time is a manifold with topology $\mathbb{R}^{3} \times \mathbb{R}$, meaning that the distances in the space-like sections parameterized by $\mathbb{R}^{3}$ are independent of the open intervals of the absolute time in $\mathbb{R}$ as dictated by the Galilean symmetry of Newton's spacetime $^{4}$. The factor $8 \pi$ comes from the spherical solid angle $4 \pi$ defined in the 3 dimensional simultaneous sections appearing in Poisson's equation $\nabla^{2} \phi=4 \pi G \rho$. Here $\rho$ is the 3 -dimensional mass density.

On the other hand, in Einstein's General Relativity the space-time manifold is parameterized by $\mathbb{R}^{4}$ without a separation between time and space as dictated by the diffeomorphism invariance of the theory. Therefore it has a different topology, which we call Lorentzian topology. In this case, once a coordinate is chosen to be the time, the diffeomorphism invariance breaks down and the Lorentz topology of the space-time changes to the product topology $\mathbb{R}^{3} \times \mathbb{R}$, the same topology of the Newtonian space-time, but with the difference that the space and time factors have different meanings ${ }^{5}$. Therefore, the change in topology in General relativity is a necessity to make the appearance of $G$ consistent with the relativistic principles.

It is also relevant to notice that the value of $G$ introduces an additional problem for General Relativity, namely the hierarchy between gravitation and the other

${ }^{a}$ We use the following notation: Greek indices run from 1 to 4 , small case Latin indices run from 5 to $D$ and large Latin indices run from 1 to $D$ and . 
fundamental (gauge) interactions. In terms of energy it is like the difference between $10^{15} \mathrm{TeV}$ to $1 \mathrm{TeV}$ in scales of energy, with nothing happening in between.

The gauge interactions start with Maxwell's equations written in the form

$$
D_{\nu} F^{\mu \nu}=4 \pi j^{\mu}, \quad D_{\nu} F^{* \mu \nu}=0
$$

where $F *$ denotes the Hodge dual of $F ; D_{\mu}=\partial_{\mu}+A_{\mu}$ and $F_{\mu \nu}=\left[D_{\mu}, D_{\nu}\right]$. A is the gauge vector-potential to be obtained by solving the above equations. Such structure is also common to the weak and strong nuclear forces. The first of these equations tells that the 3 -form or covariant antisymmetric tensor in the left hand side must equal to the current one form or covariant vector in the right hand side. This is possible only in four dimensions and consequently, the existence of the gauge fundamental interactions are consistent only in four-dimensional space-times.

Einstein's gravitation is not a gauge theory for several reasons: it does not have the same dual property; the main variable of a gauge theory is the connection $A$ determined in the adjoint representation of the gauge group, whereas in Einstein's gravity the connection is postulated. Finally, the Einstein-Hilbert action is linear in the Ricci scalar, while the actions of gauge fields are quadratic in the curvature. Thus, in Einstein's theory, the gravitational field does not possess the same four-dimensional constraint, meaning that gravitation may propagate along extra dimensions in excess of four, but it also propagate in a space-time subspace of some higher-dimensional space.

In spite of such conceptual differences, the co-existence between gravitation and gauge fields (and even their unification) can be solved without modifying General Relativity. Indeed, using the same previous arguments to justify the presence of $G$ in Einstein's equations, the coupling constant between gravitation and matter may vary depending on the number $N$ of extra dimensions accessible by gravitation. Suppose we have a space-time $V_{4}$ embedded in a Riemannian geometry of $D=$ $4+N$ dimensions with the Lorentz topology $\mathbb{R}^{D}$. By fixing $N$ extra dimensions, that topology changes to the product topology $\mathbb{R}^{4} \times \mathbb{R}^{N}$. Since the total number of space dimensions accessible by gravitation is now greater than three, the gravitational constant cannot be the same as the Newtonian $G$. The new value $G^{*}$ must be established experimentally, in cosmology and in high energy experiments ${ }^{8}$.

Admitting that the definition of the metric is the same for both spaces (an isometric embedding), the metric of the embedding space must also follow from the Einstein-Hilbert principle

$$
\frac{1}{G_{*}} \frac{\delta}{\delta \mathcal{G}_{A B}} \int{ }^{D} \mathcal{R} \sqrt{\mathcal{G}} d^{D} v=0
$$

where ${ }^{D} \mathcal{R}$ denotes the higher-dimensional scalar curvature. We Obtain the Ddimensional Einstein's equations

$$
R_{A B}-\frac{1}{2} R \mathcal{G}_{A B}=G_{*} T_{A B}, \quad A, B=1 . . D
$$

where $T_{A B}$ denotes the energy-momentum tensor of the known material sources. The projection of these equations in the four-dimensional space-times is obtained 
simply by writing them in the Gaussian coordinate system defined by the embedded space-time and the $D-4$ unit normal vector fields. We obtain in general three sets of equations involving the metric $g_{\mu \nu}$, the extrinsic curvatures $k_{\mu \nu a}$ and the third fundamental form (or torsion vector) $A_{\mu a b}$ :

$$
\begin{aligned}
R_{\mu \nu}-\frac{1}{2} R g_{\mu \nu}-Q_{\mu \nu} & =G T_{\mu \nu} \\
k_{a \mu ; \rho}^{\rho}-h_{a, \mu}+A_{\rho c a} k_{\mu}^{\rho c}-A_{\mu c a} h^{c} & =-2 \alpha_{*}\left(T_{\mu a}^{*}-\frac{1}{N+2} T^{*} g_{\mu a}\right) \\
S_{a b}-S g_{a b}-\frac{1}{2}\left[R-K^{2}+h^{2}\right] g_{a b} & =\alpha_{*} T_{a b}^{*}
\end{aligned}
$$

where we have denoted $S_{a b}=\mathcal{R}_{A B} \eta_{a}^{A} \eta_{b}^{B}$ and $S=g^{a b} S_{a b}$ and

$$
Q_{\mu \nu}=g^{a b} k_{\mu a}^{\rho} k_{\rho \nu b}-g^{a b} h_{a} k_{\mu \nu b}-\frac{1}{2}\left(K^{2}-h^{2}\right) g_{\mu \nu}
$$

It can be seen by direct calculation that $Q^{\mu \nu}{ }_{; \nu}=0$, so that $Q_{\mu \nu}$ can be associated with an observable in space-time.

The embedding itself is given by a map $X: V_{4} \rightarrow V_{D}$, satisfying the embedding equations $X^{A}{ }_{, \mu} X^{B}{ }_{, \nu} \mathcal{G}_{A B}=g_{\mu \nu}, X^{A}{ }_{, \mu} \eta^{B} \mathcal{G}_{A B}=0, \eta^{A} \eta^{B} \mathcal{G}_{A B}=1$, where $\eta_{a}^{A}$, $a=1 . . N$ are the components of the $N$ unit normal vectors to the space-time. The variations of these normals when their foot are displaced in $V_{4}$ are expressed in terms of two coefficients, the extrinsic curvature $k_{\mu \nu a}$ and the torsion vector $A_{\mu a b}$ appearing in

$$
\eta_{a, \mu}^{A}=-k_{a \mu}^{\nu} X_{, \nu}^{A}+A_{\mu a}^{b} \eta_{b}^{A}, \quad k_{\mu \nu a}=k_{\nu \mu a}, \quad A_{\mu a b}=-A_{\mu b a}
$$

Together, $g_{\mu \nu}, k_{\nu \mu a}$ and $A_{\mu a b}$, define the geometry and topology of the space-time. The integrability conditions for the above equations are given by the components of the Riemann tensor of the embedding space evaluated in the Gaussian frame of $V_{4}$, known as the Gauss-Codazzi-Ricci equations ${ }^{6}$.

$$
\begin{aligned}
& { }^{D} \mathcal{R}_{A B C D} \mathcal{Z}_{, \alpha}^{A} \mathcal{Z}_{, \beta}^{B} \mathcal{Z}_{, \gamma}^{C} \mathcal{Z}_{, \delta}^{D}=R_{\alpha \beta \gamma \delta}-2 g^{m n} k_{\alpha[\gamma m} k_{\delta] \beta n} \\
& { }^{D} \mathcal{R}_{A B C D} \mathcal{Z}_{, \alpha}^{A} \eta_{b}^{B} \mathcal{Z}_{, \gamma}^{C} \mathcal{Z}_{, \delta}^{D}=k_{\alpha[\gamma b ; \delta]}-g^{m n} A_{[\gamma m b} k_{\alpha \delta] n} \\
& { }^{D} \mathcal{R}_{A B C D} \eta_{a}^{A} \eta_{b}^{B} \mathcal{Z}_{, \gamma}^{C} \mathcal{Z}_{, \delta}^{D}=-2 g^{m n} A_{[\gamma m a} A_{\delta] n b}-2 A_{[\gamma a b ; \delta]}-g^{m n} k_{[\gamma m a} k_{\delta] n b}
\end{aligned}
$$

These equations represent the Frobenius expression for the curvature associated with a given connection and they are therefore the integrability conditions for the embedding and as such they are not dynamical equations.

Notice that in the above considerations all principles of General Relativity remain valid, so that Einstein's theory remains the same. The Einstein's equations are here seen as a consequence of the Einstein-Hilbert principle.

\section{Cosmology}

In the following, we consider the example of $D=5$ with only one time dimension and ${ }^{D} \mathcal{R}_{A B C D}=0$. This is sufficient to embed the standard model of the universe 
as proven by the analysis of the Gauss-Codazzi equations. We also consider that all material sources of gravitation are made of ordinary matter, interacting with gauge fields, so that they are also confined to the space-time. Then equations (8) is an identity and (6)-(7) reduce to

$$
\begin{aligned}
& R_{\mu \nu}-\frac{1}{2} R g_{\mu \nu}-Q_{\mu \nu}=G T_{\mu \nu}^{*} \\
& k_{\mu a ; \rho}^{\rho}-h_{a, \mu}=0
\end{aligned}
$$

The propagation of gravitation along the extra dimensions generates a continuous family of new metric geometries. To see this, consider a point in an originally given embedded space-time given by the coordinate $X^{A}$, and its (continuous) propagation on the extra dimension $\eta$ with parameter (coordinate) $y$. The curve determined by that point and with tangent vector $\eta$ gives a new point of the embedding manifold described by coordinates $Z^{A}$. For small displacements $\delta y$, the result is given by the Lie transport of $X^{A 7}$

$$
Z^{A}=X^{A}+\delta y £_{\bar{\eta}} X^{A}
$$

Repeating the same for all points in a neighborhood of $p$ we obtain congruence of curves (the orbits of $p$ ), which characterizes a one-parameter group of diffeomorphisms in the embedding space. In each of these curves, the extrinsic curvature plays the role of the Frenet acceleration in the theory of curves in space, orthogonal to the tangent vector and pointing towards its center of curvature. These points define a new embedded manifold withe metric $G_{\mu \nu}^{\prime}$ and extrinsic curvature $k_{\mu \nu}^{\prime}$ provided they satisfy similar embedding equations

$$
Z_{, \mu}^{A} Z_{, \nu}^{B} \mathcal{G}_{A B}=g_{\mu \nu}, \quad Z^{A}{ }_{, \mu} \eta^{B} \mathcal{G}_{A B}=0, \quad \eta^{A} \eta^{B} \mathcal{G}_{A B}=1
$$

Replacing $Z_{, \mu}^{A}$ in (16) we obtain the metric and extrinsic curvature of the "deformed manifold" with geometry given by

$$
\begin{aligned}
& g_{\mu \nu}^{\prime}=g_{\mu \nu}-2 y k_{\mu \nu}+y^{2} g^{\rho \sigma} k_{\mu \rho} k_{\nu \sigma} \\
& k_{\mu \nu}^{\prime}=k_{\mu \nu}-2 y g^{\rho \sigma} k_{\mu \rho} k_{\nu \sigma}
\end{aligned}
$$

Deriving the first equation with respect to $y$, we obtain

$$
k_{\mu \nu}^{\prime}=-\frac{1}{2} \frac{\partial g_{\mu \nu}^{\prime}}{\partial y}
$$

indicating that the gravitational field defined by (14)-(15) propagates in the extra dimension, as specified by the extrinsic curvature.

Three particular solutions of (15) correspond to the cases where gravitation propagates only in the four-dimensional space-times: (1) When $k_{\mu \nu}=0$ in all directions, then (14) becomes identical to Einstein's equations without topological considerations. In this case, all usual solutions of the intrinsic General Relativity are reproduced, including the Minkowski space-time taken as the ground state of the gravitational field. (2) When $k_{\mu \nu}=\varphi(x) g_{\mu \nu}$ in all directions. In this case, we obtain Einstein's theory with an scalar field $\varphi$. The space-time has an umbilicus point at 
$x$. (3) Finally, if $k_{\mu \nu}=\alpha_{0} g_{\mu \nu}$ where $\alpha_{0}$ is constant, then the space-time is a space with constant curvature ${ }^{6}$. Replacing this in (9), we obtain Einstein's equations with a cosmological constant, $-\Lambda=3 \alpha^{2}$, so that $\Lambda$ appears as a very particular deformation of the space-time.

For the standard FLRW cosmology, Friedmann's equation is replaced by ${ }^{9}$

$$
\frac{\dot{a}^{2}}{a}+\frac{\kappa}{a^{2}}=-\frac{8 \pi G}{3} \rho+\frac{b^{2}}{a^{4}}, \quad \text { with } \quad \frac{\dot{a}}{a} \propto \frac{\dot{b}}{b}
$$

where we have denoted $b(t)=k_{r r}$, the radial extrinsic curvature and $\kappa$ is the intrinsic curvature of the space sections as given by the sum of the internal angles of a triangle.

We have compared this result with the Turner-White phenomenological fluid (x-fluid), and later, using the model independent distance-measurement statistical analysis, we have found that the term $b^{2} / a^{4}$ fits the intervals compatible with the acceleration of the universe. The acceleration of the universe is given by the radial component of the extrinsic curvature $k_{r r}$, and the last term in (19) describes the radiation energy resulting from the stretch of the geometry as it expands in the vacuum.

It is interesting to notice that the space sections are flat in the sense that $\kappa=0$, but they are not like flat planes because they have non-zero radial components $k_{r r}$. The case of $k_{\mu \nu}=\propto g_{\mu \nu}$ corresponds to the cosmological constant. It does not occur here because the acceleration would be exclusively in the time direction and not in the space-sections. Such qualitative difference may in principle be detected by a detailed analysis of the CMB power spectrum.

\section{References}

1. Ya. B. Zel'dovich and I. D. Novikov.

The Structure and Evolution of the Universe. Chicago U. P. Chicago (1983).

2. S. Weinberg, Reviews of Modern Physics. 61, 1, (1989).

3. S. Weinberg. e-Print: astro-ph/0005265

4. M. D. Maia Geometry of the Fundamental Interactions. Springer, New York (2010).

5. G. Gibbons, Topology Change in Classical and Quantum Gravity, arXiv: 1110.0611.

6. L. P. Eisenhart, Riemannian Geometry, Princeton U.P. Princeton (1966).

7. M. Crampin and F.A. E. Pirani, Applicable Differential Geometry, Cambridge U.P. Cambridge U.K. (1986).

8. N. Arkani-Hamed et al., Phys. Lett. B429, 263 (1998).

9. M. D. Maia, E.M. Monte J.M.F. Maia and J.S. Alcaniz, Class. Quant. Grav. 22, 1623 (2005).

10. M. Maia, J. Alcaniz,E. M. Monte and A. Capistrano arXiv:1101.3951 [gr-qc] 